\title{
Antibody Levels in COVID-19 Affected by Age, Gender, and Disease
}

Heeral Patel

Editorial Assistant

Citation: EMJ Hematol. 2021;9[1]:32-34.

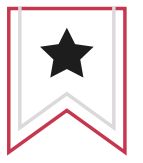

\begin{abstract}
T THE EUROPEAN Hematology Association (EHA) 2021 Virtual Congress, Evangelos Terpos, University of Athens School of Medicine, Greece, discussed COVID-19 and how neutralising antibody levels are affected by various factors such as age, gender, and disease.
\end{abstract}

The COVID-19 pandemic has been a catalyst for rapid vaccine development by scientists and researchers around the globe compared to the usual time frame. From bench to bedside, the process of drug development can take up to 10 years; however, a global effort that involved pharmaceutical companies working collaboratively with international researchers has significantly accelerated the process. Despite the vaccine's high efficacy, there is still much more to learn about how individual groups respond to the vaccine.

\section{"Not only were neutralising} antibodies higher just before the second dose but they were much higher for the younger age group, aged 25-50 years, compared to the octogenarians, aged 80-95 years. "
Terpos shared his thoughts regarding vaccination against COVID-19. Firstly, Terpos discussed the differences in efficacy between the most popular mRNA vaccines: BNT162B2 (Pfizer-BioNTech), mRNA-1273 (Moderna), AZD1222 (AstraZeneca), and Ad26.COV2.S (Johnson \& Johnson). The vaccines by Pfizer-BioNTech and Moderna have the highest efficacy of $>90 \%$, whereas the latter two have a slightly lower efficacy rate: $70-90 \%$.

\section{AGE- AND GENDER-DEPENDENT ANTIBODY RESPONSES}

In light of this observation, Terpos expressed the importance of testing the vaccine in healthy populations. He shared his research from a recent study looking at age-dependent and gender-dependent antibody responses after the Pfizer-BioNTech vaccine. Interestingly, the groups involved in this study were the first to get vaccinated in Greece. 
Firstly, Terpos recruited 225 health workers of a median age of 49 years and volunteering octogenarians of 85 years median age. The team measured the neutralising activity of antibodies at different stages of vaccination: one day before the vaccine (Day 1), eight days after the first dose, two weeks after the second dose, and four weeks after the second dose. Results showed that the two groups had no neutralising activity before the first dose and almost the same results 8 days after the first dose.

Interestingly this changed just before the second dose was administered. Data presented

"Antibody levels were

initially higher; however,

after Day 22 the levels were

very similar in both cohorts,

with a slight favour towards

the Pfizer-BioNTech vaccine

with a p value of $0.03 . "$

by Terpos demonstrated that, not only were neutralising antibodies higher just before the second dose but they were much higher for the younger age group, aged 25-50 years, compared to the octogenarians, aged 80-95 years. The neutralising antibody levels changed yet again after the second dose, with almost all groups showing very high antibody levels. Taken together, these results confirm that for those aged 50 years and above, the second dose is necessary. Intriguingly, neutralising antibody levels were also affected by gender. In the elder age group, females developed significantly more antibodies than males on Day 22 and Day 50. The results suggest that the levels of antibodies are gender-dependent in older individuals. The same was not concluded for the younger age group because, although antibody levels were higher in females, the results were not statistically significant.

Terpos shared unpublished data showing the results from this cohort after 90 days postsecond dose. The data confirmed that although there was a slight decrease in neutralising antibodies compared to Day 36 and Day 50, no patient was recorded with antibody levels $<30 \%$, the level at which a person is considered COVID19-positive. To better understand the significance of these results and the main factors for antibody production, the team used a machine learning analysis approach. The results showed that age is a negative factor to neutralising antibody levels in older age groups, confirming that younger individuals produced higher antibody levels. Terpos also looked at BMI but found that this had no impact in the levels of antibody; however, in previous studies, BMI has been shown to have a negative impact on antibody levels.

\section{ANTIBODY LEVELS IN INDIVIDUALS PREVIOUSLY INFECTED WITH COVID-19}

Following on from the initial research, it is vital to understand why the neutralising antibody levels on Day 1 were higher in some groups even before the first dose. Terpos believes this is because either they had previously been infected with COVID-19 or they had COVID-19 at the time without realising. After the first dose, the data showed that the antibody levels increased to a very high level and stayed that way even at Day 22. This suggests that this previously infected population may not need a second dose.

To comprehend why this may be, the levels of inflammatory cytokines were measured in the different groups. Those who had high neutralising antibodies on Day 1 also had high inflammatory cytokines on Day 2 (the day after the first dose), whereas those who did not have high levels of antibodies on Day 1 only showed a high inflammatory response after the second dose. Again, this reinforces the argument that those who had already been infected and had high neutralising antibodies may not need a second dose.

\section{THE EFFECT OF HAEMATOLOGICAL MALIGNANCIES ON ANTIBODY LEVELS}

An interesting question was raised by Terpos and his team as to whether there is a difference in neutralising antibody levels between the two most common vaccines distributed in Europe currently: BNT162B2 and AZD1222.

Antibody levels were initially higher in the PfizerBioNTech vaccine cohort; however, after Day 22 the levels were very similar in both cohorts, 
